\title{
SPONTANEOUS SMALL-SCALE MELT SEGREGATION IN PARTIAL MELTS UNDERGOING DEFORMATION
}

\author{
David J. Stevenson
}

\section{Division of Geological and Planetary Sciences, California Institute of Technology}

\begin{abstract}
A partial melt undergoing large scale deformation is shown to be unstable with respect to small scale redistribution of melt, provided the shear viscosity of the matrix depends on melt fraction. In the physically realistic case where melt "softens" the matrix, melt migrates along the direction parallel to the axis of minimum compressive stress and accumulates in "veins" (melt-rich lenses). The maximum growth rate of the instability is of order $-\dot{\epsilon}_{o} d \ln \eta / d f$ ( $\dot{\epsilon}_{o} \equiv$ largest component of the principal strain tensor, $\eta=$ shear viscosity, $f=$ melt fraction), and plausibly fast enough to invalidate the conventional applications of Darcy's law (e.g., mid-ocean ridges, subduction zones) since melt migrates into veins in preference to being pervasively flushed vertically. The preferred lengthscale of the instability is poorly determined but probably $\sim$ meters. Veins may eventually form an interconnected drainage network, allowing rapid vertical flushing of melt.
\end{abstract}

\section{Introduction}

There has been much activity in recent years on the problems of melt migration, both from geophysical and geochemical perspectives. In almost all of this work, it is assumed that there exists a network of interconnected melt tubules which is spatially homogeneous on lengthscales large compared to an individual grain diameter yet small compared to geological (e.g., kilometer) scales. This forms the basis of applying Darcy's law to a variety of melt migration problems, the best example being the formation of oceanic crust at spreading centers (e.g., Turcotte, 1982). Although recent work has elaborated on the theory (e.g., McKenzie, 1987), and the role of large scale motions (Scott, 1988; Scott and Stevenson, 1989), the validity of the underlying assumptions has remained largely unchallenged.

However, there is good reason to doubt that Darcy's law is a sufficient description. Geologic evidence of dikes and veins in mantle peridotites (Nicolas and Jackson, 1982; Nicolas, 1986) suggest some process of melt localization at depth. Sleep (1988) discusses this evidence and describes how veins and dikes can tap the melt by porous flow, but offers no quantitative explanation for the origin of veins. Laboratory evidence (Beeman and Kohlstedt, 1987) also suggests smallscale melt segregation in olivine-melt aggregates. Geophysical modeling has also cast doubt on uniformly pervasive, percolative flow of melt: Scott and Stevenson (1989) find that the concentration of crustal production at mid-ocean ridge crests is best achieved in circumstances where melt retention

Copyright 1989 by the American Geophysical Union.

Paper number $89 \mathrm{GL} 01416$.

0094-8276/89/89GL-01416\$03.00 by the mantle is initially higher (i.e., very low permeability), followed by a rapid, concentrated release of melt. Tentatively, the emerging picture is one in which the melt does not percolate as fast as conventional theory would suggest until some kind of "drainage network"' is established.

The model developed below is a fluid dynamical instability. Before proceeding to an explanation of its origin. I briefly consider three other possibilities: (1) melt pocket formation driven by surface tension; (2) lateral variability of the source rock; and (3) brittle failure. None can be excluded as important but none is as universal as the mechanism proposed here.

(1) Surface tension affects can arise in two ways. If the dihedral angle exceeds $60^{\circ}$ but some permeability exists, then the melt fraction satisfies a nonlinear diffusion equation in which the diffusivity is negative (Stevenson, 1986). This implies a spontaneous amplification of any small variation of melt fraction. However, the dihedral angle for basaltic melt in contact with olivine grains is $\sim 30^{\circ}$ (Waff and Bulau, 1979). Partially molten peridotite is more complicated because of the pyroxenes (Toramaru and Fujii, 1986), but our current understanding of the surface tension effects does not indicate spontaneous melt segregation in peridotite at realistic melt fractions.

(2) Lateral variability of the source rocks has been invoked to explain some isotopic and elemental variations in erupted basalts (Prinzhofer et al., 1989; Holness and Richter, 1989). However, small scale lateral variability in melting rate (and thus melt fraction) should be damped by surface tension driven diffusion (Stevenson, 1986), if no other mechanism (such as that described below) is present.

(3) The idea of brittle failure is perhaps the most attractive of all, yet seems exceedingly difficult in the mantle because the primary response to imposed strain is creep. Even at a deviatoric stress equal to the low failure strength of partially molten rock (plausibly as low as a few hundred bars), the equilibrium strain rate due to creep is likely to be $10^{-10} \mathrm{~s}^{-1}$ or more, fast by geologic standards. Nicolas (1986) proposes that failure can occur because of the difference in hydrostatic heads that builds up between a rigid (noncompacting) matrix and the permeating immobile, interconnected fluid. This stress difference is $\sim \Delta \rho g H$, where $\Delta \rho$ is the density difference between solid and melt and $H$ is the height of the column. But the matrix cannot be rigid (it compacts) and the melt consequently cannot be immobile if it is interconnected, irrespective of boundary conditions. The maximum stress in these circumstances is $\sim \Delta \rho g L$ where $L$ is the compaction length (McKenzie, 1987) and is $\sim$ few bars, inadequate for failure. Conceivably, brittle failure occurs later in the evolution (after veins have formed) or in the short timescale nearsurface emplacement of ophiolites, where the field evidence certainly suggests a failure phenomenon (Sleep, 1988). 


\section{The Basic Model}

Consider a partially molten rock undergoing twodimensional deformation. Choose an $x, z$ coordinate system, coincident with the principal stress axes, in which the center of mass velocity $C$ of a two-phase element satisfies

$$
\begin{aligned}
& \frac{\partial C_{x}}{\partial x}=\dot{\epsilon}_{o}=-\frac{\partial C_{z}}{\partial z}>0 \\
& C=(1-f) V_{s}+f L_{\ell}
\end{aligned}
$$

where $\dot{\epsilon}_{o}$ is constant, $f$ is the melt fraction, and $V_{s}, V_{\ell}$ are solid and liquid phase mean velocities, respectively. I assume $f \ll 1$. For the present, ignore gravity, surface tension, and any variation in the $z$-direction. If there is no melt migration, then force balance requires that the stress component $\sigma_{x x}$ does not vary in the $x$-direction, where

$$
\sigma_{x x}=-P_{\ell}+2 \eta \dot{\epsilon}_{o}
$$

The liquid pressure is $P_{\ell}$ and the matrix shear viscosity is $\eta$. Now suppose that there exists some small spatial fluctuation in melt fraction along the $x$-direction. In the physically reasonable case where low $f$ corresponds to high $\eta$ and vice-versa (Cooper and Kohlstedt, 1986), constancy of the $\sigma_{x x}$ implies that $P_{t}$ must be low where $f$ is high and vice versa. But melt will flow into low pressure regions, so melt accumulates where it is already concentrated: This is the instability. We can even estimate the growthrate of this instability by considering the limit in which the wavelength of the melt fraction fluctuations is so small that fluctuations in $P_{\ell}$ can be immediately eliminated by porous flow. Equation (3) now becomes (Scott, 1988)

$$
\sigma_{x x}=-P_{\ell}+2 \eta \dot{\epsilon}_{o}-\left(\varsigma+\frac{4}{3} \eta\right) \frac{\partial S_{x}}{\partial x}
$$

where $\zeta$ is the bulk viscosity, and $S_{x}$ is the $x$-component of the "segregation" $S$ defined as

$$
S \equiv C-V_{s} \equiv f\left(V_{\ell}-{\underset{\sim}{s}}_{s}\right)
$$

If $P_{\ell}$ is constant then low $\eta$ (high $f$ ) must correspond to negative $\partial S_{x} / \partial x$. However, continuity requires

$$
\frac{\partial f}{\partial t}=-\frac{\partial S_{x}}{\partial x}
$$

for small $f$, neglecting advection. By setting $\partial \sigma_{x x} / \partial x=0$, I finally obtain

$$
\begin{gathered}
\frac{\partial}{\partial t}\left(\frac{\partial f}{\partial x}\right)=\sigma_{m}\left(\frac{\partial f}{\partial x}\right) \\
\sigma_{m} \equiv 2 \alpha \beta \dot{\epsilon}_{o} \\
\alpha \equiv \frac{\eta}{\left(\zeta+\frac{4}{3} \eta\right)} \\
\beta \equiv-\frac{d \ln \eta}{d f} .
\end{gathered}
$$

\section{Detailed Analysis}

I continue to assume 2-D flow, $f \ll 1$, and lengthscales small enough that I can treat $f$ and the large scale flow as spatially uniform in the basic state (i.e., in the absence of the instability). I can always define an $x, z$ coordinate system as above, but gravity $g$ is allowed to have an arbitrary relative orientation. The equations are in the form presented by Scott (1988), except that $\eta$ and $\zeta$ are allowed to be variable; the widely cited equations of McKenzie (1984) are entirely equivalent. The surface tension effect (Stevenson, 1986) is also included. Accordingly, Darcy's law becomes

$$
\begin{gathered}
S=\frac{k(f)}{\eta_{\ell}}\left[-q \Delta \rho-\frac{\gamma}{f^{3 / 2}} \underset{\sim}{\sim} f+\underset{\sim}{\sim}\left(\left[\zeta-\frac{2}{3} \eta\right] \underset{\sim}{\nabla}\right)\right. \\
-\underset{\sim}{\nabla} \times(\eta \underset{\sim}{\nabla} \times[S-C])]
\end{gathered}
$$

where $k$ is the permeability, $\eta_{\ell}$ is the liquid viscosity, $\Delta \rho$ is the density difference between solid and liquid, and $\gamma$ is of order the solid-liquid surface tension divided by a grain diameter. The basic state is just the first term with $k$ constant. For computational convenience, I choose a local frame of reference in which the large scale flow $C \simeq Q$; this enables me to ignore advection, an effect that tums out not to inhibit the instability (except, of course, when the material is advected to the boundaries or to a subsolidus region). I consider a perturbation in the melt fraction of the form

$$
f=f_{o}+f^{\prime} \sin (q x+w t) e^{\sigma t}
$$

where $f^{\prime} \ll f_{r}$; all quantities are real. Variations with $z$ are omitted. Rapid variations in the $z$-direction can destroy the instability because the $\sigma_{z z}$ stress acts to squeeze melt out of regions where melt is concentrated, but slow variations in the $z$-direction have little effect of the instability except for the caveat given below. It is sufficient that the $z$-extent of the melt lens be large compared to its width. Upon performing standard linear analysis on eq. (11), I determine that

$$
\begin{gathered}
\sigma=\frac{q^{2} L^{2}\left(\sigma_{m}-\sigma_{s}\right)}{\left(1+q^{2} L^{2}\right)} \\
\omega=\left(q g_{i} \Delta \rho \frac{d k}{d f}\right) / \eta_{\ell}\left(1+q^{2} L^{2}\right) \\
\sigma_{s} \equiv \frac{\gamma}{\eta f_{o}^{3 / 2}} \\
L \equiv\left(\frac{k\left[\zeta+\frac{4}{3} \eta\right]}{\eta_{\ell}}\right)^{1 / 2} .
\end{gathered}
$$

The lengthscale $L$ is often referred to as the compaction length. Equation (13) shows that the instability exists provided $\sigma_{m}>\sigma_{s}$ and is fastest as $q L \rightarrow \infty$. Some cautionary comments should be made before applying the results:

1. The strain tensor is assumed locally uniform (eq. 1). This does not apply to all situations, especially laboratory experiments. It is a reasonable approximation beneath midocean ridges, where the flow is imposed by the boundaries.

2. Although gravity does not appear to play a role in the existence of the instability, it can "short circuit" the instability when $q L$ is not large, by allowing rapid flow in the $z$-direction, away from the melt fraction enhancements.

3. The model is meaningless if $q \gtrsim 1 / R$, where $R$ is a grain radius, since the continuum approximations implicit in 
the fundamental equations fail. In fact, if I adopt the view that all variables should be averaged over a grain and its immediate neighbors and use only averaged variables in the differential equations, then there is a finite $q \sim(L R)^{-1 / 2}$ at which $\sigma$ is a maximum. For $L \sim 100$ meters, $R \sim 1 \mathrm{~cm}$, the preferred lengthscale of the instability is $\sim 1$ meter.

4. Fluctuations in $f$ cause fluctuations in the buoyancy force available to drive $C$. The above analysis ignores these small scale components of the mean flow. This can be shown to be a valid approximation for $f \ll 1$, or $q L \gg 1$.

\section{Application}

There are two issues: (1) Is $\sigma_{m}>\sigma_{q}$ ? (2) If so, is the growthrate fast enough to be geologically important? The first question can be restated in terms of a critical melt fraction $f_{c}$ above which $\sigma_{m}>\sigma_{n}$. Cooper and Kohlstedt (1986) suggest that their theology data are consistent with a model in which the strain rate is determined by the size of the zone of contact between adjacent grains. It would then follow that $\eta \simeq \eta_{o}\left(1-A f^{1 / 2}\right)$; their data imply $A \simeq 3$ or 4 at small $f$. This suggests that $\beta \approx 3.5 / f_{o}^{1 / 2}$. Accordingly, $\sigma_{m}>\sigma_{s}$ becomes

$$
f_{o}>f_{c} \equiv \frac{\gamma}{7 \eta \dot{\epsilon}_{o} \alpha} \sim \mathbf{1 0}^{-3} .
$$

which is easily satisfied.

To achieve a strong effect from this instability, $\sigma_{m} T \gg 1$ is required, where $T$ is the "available time." In mantle upwelling beneath mid-ocean ridges, $T$ is of order the time for mantle material to rise some substantial fraction of the distance between the solidus and the surface. I estimate $\sigma_{m} T \sim 3$ to 10 and the instability can be well developed. The parameters are uncertain, however, and more laboratory data on the rheology of partial melts would be very helpful for assessing this phenomenon.

In the limit $q L \gg 1$, the equation governing the instability can be integrated:

$$
\begin{aligned}
\frac{d f}{d t} & =\phi(f)+\chi(t) \\
\phi^{\prime}(f) & \equiv \sigma_{m}(f)-\sigma_{s}(f)
\end{aligned}
$$

and $\chi(t)$ is chosen so that the total melt budget is correct, i.e.,

$$
\left\langle\frac{d f}{d t}\right\rangle=\left.\frac{\partial f}{\partial t}\right|_{\text {melt }}
$$

where the brackets denote a spatial average and the RHS of the equation represents newly generated melt, for example by pressure release upwelling. I can define a dimensionless number $P=\sigma_{m} /(\partial \ln f / \partial t)_{\text {melt }}$ and there will be a critical value of $P=P_{c}$ above which the melt concentrates in veins. Numerous numerical experiments were performed, but $P_{\mathrm{c}}$ is sensitive to initial conditions. As an example, $P_{c} \sim 5$ in experiments where $\langle f\rangle=0.01$ initially, with a $10 \%$ white noise fluctuation and spatially uniform melt production. Figure 1 is an illustration of the outcome of one such experiment.

\section{Interpretation}

Consider mantle material undergoing pressure release melting as it rises. Deviatoric stress is generally present and

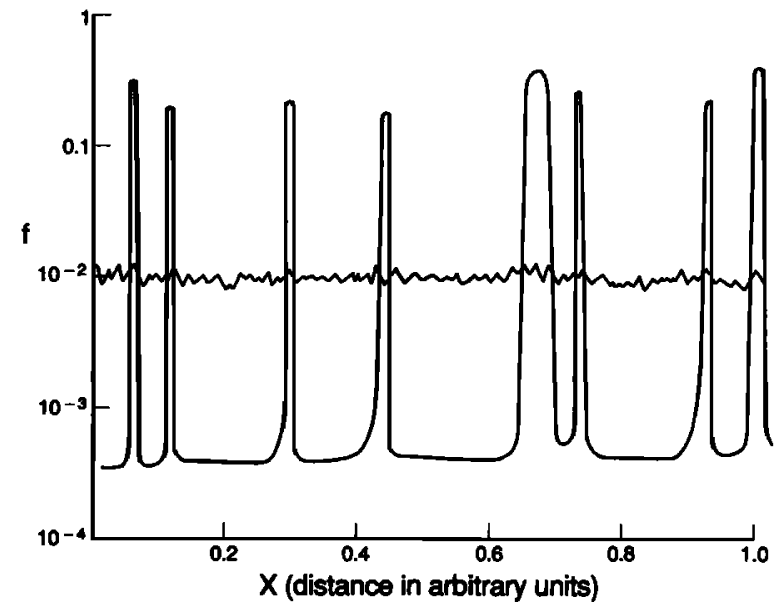

Fig. 1. Numerical solution of equation (16), for an initial condition consisting of $1 \%$ uniform melt fraction and a small white noise perturbation. The melt fraction is $f$ and $X$ is in the direction of extension (i.e., $\sigma_{x, r}$ is the minimum principal compressive stress component). Both the initial condition and the endpoint of the simulation are shown. In this case, additional melt is continually supplied uniformly in space, so that an additional $2 \%$ is added during the simulation. The elapsed time corresponds to roughly ten $e$-folding times of the initial (linear) instability. Notice the development of "veins" and the almost total absence of melt between veins despite continuing replenishment. Notice also that the number of veins is small compared to the high frequency of the initial "noise" because veins "ripen" and sometimes merge as they develop. The horizontal scale is arbitrary but presumably $\sim$ meters.

the proposed instability will generally occur faster than the upwelling time, causing new melt to channel into veins rather than be flushed vertically. These veins can develop further in a way described by Sleep (1988), whose analysis can be considered as a special or finite amplitude case of what is presented here. It is likely (though not proved here) that the veins initially lack contiguity over geologic distances in the directions perpendicular to the minimum compressive stress direction thereby greatly reducing the effective permeability of the medium. The lack of contiguity seems plausible, because the precise placement of veins in the $x$-direction is random (cf. Figure 1) and is likely to fluctuate as each new batch of material rises. One then has the prospect of explaining the puzzles mentioned in the introduction.

\section{Conclusion}

Partially molten rock undergoing deformation is texturally unstable because of small scale redistribution of melt relative to the solid. Melt aggregates into veins because these are low pressure regions. The preferred lengthscale of this instability is poorly determined but is certainly small compared to the compaction length $(\sim 100$ meters $)$ and large compared to grains. A lengthscale $\sim$ meter is plausible. This phenomenon invalidates the conventional application of Darcy's law, in which the permeability is associated with a pervasive, microscopic network of melt tubules. Further work is 
needed to establish the topology and architecture of the melt drainage network implied by the instability.

Acknowledgements. Comments from N. Sleep and an anonymous reviewer were helpful. This work is supported by NSF Earth Sciences grants EAR8618511 and EAR8816268. Contribution number 4764 from the Division of Geological and Planetary Sciences, California Institute of Technology, Pasadena, Califomia 91125.

\section{References}

Beeman, M. L., and D. L. Kohlstedt, Deformation of olivinemelt aggregates at high temperatures and confining pressures. EOS 68, 1453, 1987.

Cooper, R. F., and D. L. Kohlstedt, Rheology and structure of olivine-basalt partial melts. J. Geophys. Res. B9, 93159324, 1986.

Holness, M. B., and F. M. Richter, Possible effects of spreading rate on MORB isotopic and rare earth composition arising from melting at a heterogeneous source. $J$. Geol. 97, 247-260, 1989.

McKenzie, D. P., The compaction of igneous and sedimentary rocks. J. Geol. Soc. (London) 144, 299-307, 1987.

McKenzie, D. P., The generation and compaction of partial melts. J. Petrol. 25, 713-765, 1984.

Nicolas, A., and M. Jackson, High temperature dikes in peridotites: Origin by hydraulic fracturing. J. Petrol. 23, 568582, 1982.

Nicolas, A., A melt extraction model based on structural studies in mantle peridotites. J. Petrol. 27, 999-1022, 1986.
Prinzhofer, A., E. Lewin, and C. J. Allegre, Stochastic melting of the marble cake mantle: Evidence from local study of the East Pacific Rise at $12^{\circ} 50^{\prime}$ N. Earth Planet. Sci. Letr. 92, 189-206, 1989.

Scott, D. R., and D. J. Stevenson, A self consistent model of melting, magma migration and buoyancy-driven circulation beneath mid-ocean ridges. J. Geophys. Res. 94, 2973$2988,1989$.

Scott, D. R., The competition between percolation and circulation in a deformable porous medium. J. Geophys. Res. 93, 6451-6462, 1988.

Sleep, N. H., Tapping of melt by veins and dikes. J. Geophys. Res. 93, 10,255-10,272, 1988.

Stevenson, D. J., On the role of surface tension in the migration of melts and fluids. Geophys. Res. Lett. 13, 1149-1152, 1986.

Toramaru, A., and N. Fujii, Connectivity of melt phase in a partially molten peridotite. J. Geophys. Res. 91, 92399252, 1986.

Turcotte, D. L., Magma migration. Ann. Rev. Earth Planet. Sci. 10, 397-408, 1982.

Waff, H. S., and J. R. Bulau, Equilibrium fluid distribution in an ultramafic partial melt under hydrostatic stress conditions. J. Geophys. Res. 84, 6109-6114, 1979.

D. J. Stevenson, Division of Geological and Planetary Sciences, California Institute of Technology, Pasadena, California 91125 .

(Received June 2, 1989;

accepted July 5, 1989.) 